\title{
Density functional study of graphene antidot lattices: Roles of geometrical relaxation and spin
}

Fürst, Joachim Alexander; Pedersen, Thomas Garm; Brandbyge, Mads; Jauho, Antti-Pekka

Published in:

Physical Review B Condensed Matter

Link to article, DOI:

10.1103/PhysRevB.80.115117

Publication date:

2009

Document Version

Publisher's PDF, also known as Version of record

Link back to DTU Orbit

Citation (APA):

Fürst, J. A., Pedersen, T. G., Brandbyge, M., \& Jauho, A-P. (2009). Density functional study of graphene antidot lattices: Roles of geometrical relaxation and spin. Physical Review B Condensed Matter, 80(11), 115117. https://doi.org/10.1103/PhysRevB.80.115117

\section{General rights}

Copyright and moral rights for the publications made accessible in the public portal are retained by the authors and/or other copyright owners and it is a condition of accessing publications that users recognise and abide by the legal requirements associated with these rights.

- Users may download and print one copy of any publication from the public portal for the purpose of private study or research.

- You may not further distribute the material or use it for any profit-making activity or commercial gain

- You may freely distribute the URL identifying the publication in the public portal 


\title{
Density functional study of graphene antidot lattices: Roles of geometrical relaxation and spin
}

\author{
Joachim A. Fürst, ${ }^{1, *}$ Thomas G. Pedersen, ${ }^{2}$ Mads Brandbyge, ${ }^{1}$ and Antti-Pekka Jauho ${ }^{1,3}$ \\ ${ }^{1}$ DTU Nanotech-Department of Micro- and Nanotechnology, Technical University of Denmark, DK-2800 Kongens Lyngby, Denmark \\ ${ }^{2}$ Department of Physics and Nanotechnology, Aalborg University, DK-9220 Aalborg East, Denmark \\ ${ }^{3}$ Department of Applied Physics, Helsinki University of Technology, P.O. Box 1100, FI-02015 HUT, Finland
}

(Received 8 April 2009; revised manuscript received 29 June 2009; published 18 September 2009)

\begin{abstract}
Graphene sheets with regular perforations, dubbed as antidot lattices, have theoretically been predicted to have a number of interesting properties. Their recent experimental realization with lattice constants below 100 nanometers stresses the urgency of a thorough understanding of their electronic properties. In this work, we perform calculations of the band structure for various hydrogen-passivated hole geometries using both spinpolarized density functional theory (DFT) and DFT based tight-binding (DFTB) and address the importance of relaxation of the structures using either method or a combination thereof. We find from DFT that all structures investigated have band gaps ranging from 0.2 to $1.5 \mathrm{eV}$. Band gap sizes and general trends are well captured by DFTB with band gaps agreeing within about $0.2 \mathrm{eV}$ even for very small structures. A combination of the two methods is found to offer a good trade-off between computational cost and accuracy. Both methods predict nondegenerate midgap states for certain antidot hole symmetries. The inclusion of spin results in a spinsplitting of these states as well as magnetic moments obeying the Lieb theorem. The local-spin texture of both magnetic and nonmagnetic symmetries is addressed.
\end{abstract}

DOI: 10.1103/PhysRevB.80.115117

PACS number(s): 73.63.Fg

\section{INTRODUCTION}

Graphene, the single-atom thick two-dimensional sheet of carbon atoms, has stimulated considerable experimental ${ }^{1}$ and theoretical research ${ }^{2}$ as well as proposals for future nanodevices. ${ }^{3}$ Various graphene-based applications have been realized in recent years ${ }^{4,5}$ and the relevance for application in devices is heavily increased by the rapidly improving ability to pattern monolayer films with electronic-beam lithography ${ }^{1}$ where features on the ten $\mathrm{nm}$ scale have been obtained. ${ }^{6,7}$ Moreover, recent advances in chemical vapor deposition of graphene (see e.g., Ref. 8) are promising for fabrication of large area, high-quality devices.

Yet another way of nanoengineering graphene consists of defining an antidot lattice on graphene by means of a regular array of nanoscale perforations. This theoretical idea (see Pedersen et al.,${ }^{9,10}$ ) who showed using tight-binding calculations that antidot lattices change the electronic properties from semimetallic to semiconducting with a significant and controllable band gap. These changes in the bandstructure can be experimentally studied by either transport or optical absorption methods. While the tight-binding calculations of Refs. 9 and 10 are a convenient way of obtaining qualitative predictions, they must be bench-marked against some $a b$ initio method, such as the density-functional theory (DFT), in order to fully assess their reliability. Such a comparison is one of the main goals of the present paper. Unfortunately, fully $a b$ initio methods become computationally very expensive as the unit cell size increases, and this is the case for perforations that can presently be achieved. Therefore, there is a great need for a computational method, which interpolates the accuracy and cost between tight-binding and DFT theories. In this paper we also investigate one such method, the DFT based tight-binding approach (DFTB).

We next briefly assess the experimental situation. Antidot lattices on graphene with lattice spacings down to $80 \mathrm{~nm}$ have recently been realized experimentally by several groups. Eroms and Weiss ${ }^{11}$ report magnetotransport measurements and clearly observe how the antidot lattice modifies the Quantum Hall effect and the weak localization behavior. The achievable mobilities for graphene on oxidized silicon substrates (as is the case in this experiment) are still too low, and the antidot lattice spacing is still too large to fully reflect the details of the band-structures we calculate. Shen et al. ${ }^{12}$ have reported commensurability oscillations in graphene flakes on $\mathrm{SiC}$ substrate, however their geometry did not allow an independent determination of the carrier density. These two experiments are important in demonstrating how the transport gap due to the antidot lattice affects magnetotransport measurements. Midgap states, as found for certain antidot lattices (see Sec. III below), would be another important diagnostics for the antidote-induced bandstructure. These states may be the cause of a metal-insulator transition, as recently revealed by an experimental study of ion-induced defects. ${ }^{13}$ Perhaps the most direct means of studying the antidot-induced gap would be to perform infrared optical absorption measurements. We are not aware of any optical experiments on antidot lattices on graphene. However, very recently an experiment addressing the optical gap in an electrically gated bilayer graphene was published. ${ }^{14}$ We have recently considered the generic features of optical absorption in gapped graphene using a phenomenological approach, ${ }^{10,15}$ and a quantitative comparison between experiments and an $a b$ initio theory for optical absorption would be most useful.

Experiments are available for several other nanostructures based on graphene. Quantum dots and graphene ribbons have been demonstrated with dimensions of only a few nm. ${ }^{16}$ Very recently, Girit et al. ${ }^{17}$ studied the dynamics at the edges of a growing hole in real time using a transmission electron microscope. Both in the experiment and in Monte Carlo simulations they find the zigzag edge formation to be the most stable structure. This is in agreement with the findings of Jia et al. ${ }^{18}$ who demonstrate a method to produce graphitic 

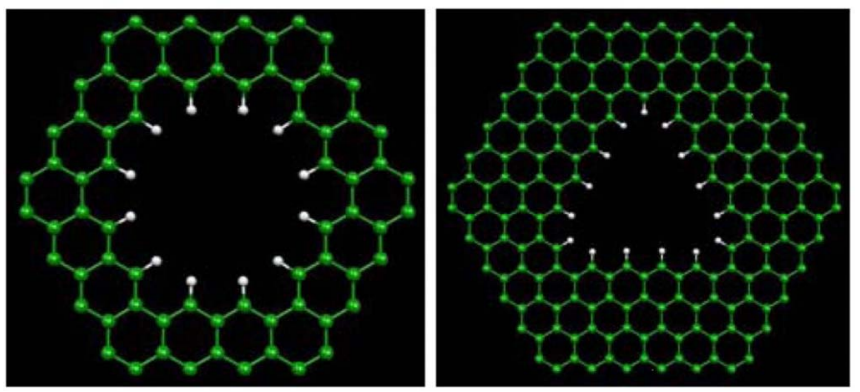

FIG. 1. (Color online) The unit cell of the $\{4,2\}_{\circ}($ left $)$ and $\{6,5\}_{\triangle}$ (right) system. The hexagonally shaped unit cells are repeated in plane to form a honeycomb lattice of antidots. The carbon atoms (green/gray) are hydrogen terminated (white) along the hole edges.

nanoribbon edges in a controlled manner via Joule heating. This opens the possibility of making antidot lattices with a desired hole geometry. Experiments of this kind are highly relevant for $a b$ initio modeling of perforations in graphene because they provide information about the microscopic structure of the edge of the perforation; this is a necessary input for any quantitative modeling.

While carbon nanotubes, grapheme, and recently graphene ribbons have been studied extensively using first principles methods, antidot lattices in graphene have mainly been treated with simpler models. ${ }^{9,19}$ The very recent work by Vanevic et al. ${ }^{19}$ uses a $\pi$-orbital tight-binding model to study antidot lattices with rather large lattice constants (these systems are more easily accessed experimentally but cannot be analyzed in terms of ab initio methods). Their focus is on the possible occurrence of midgap states without introducing defects in the antidot lattice, as was the case in the original proposal by Pedersen et al. ${ }^{9}$

Many studies on magnetization have been reported for various graphene structures. ${ }^{20-27}$ The origin of the magnetism can be understood based on the theory by Lieb, ${ }^{20}$ and the subsequent related work by Inui et $a l^{28}$ on the properties of the bipartite lattice. Single vacancies and their spin properties have been studied by, e.g., Lehtinen et al. ${ }^{21}$ and Palacios et $a l . ; 2$ the latter paper also investigates voids in both graphene and graphene ribbons in detail using a mean-field Hubbard-model. Magnetization has also been studied in carved slits, ${ }^{23}$ finite ribbons ${ }^{24}$ and flakes ${ }^{24,25}$ as well as rings ${ }^{29}$ and notches. ${ }^{30}$ Recently, DFT treatments of magnetic properties of nanoholes in graphene ${ }^{31}$ and graphene films ${ }^{32}$ have been published.

The realized antidot lattices with hole sizes of several tens of nanometers and even larger lattice spacings involve several thousands of atoms in a unit cell and are computationally too costly to be treated with DFT in a systematic manner. The DFT based tight-binding method, DFTB, ${ }^{33}$ however, allows one to address such large systems. The difference in computational cost between DFT and DFTB is for the present study found to be at least a factor of thirty. We, thus, investigate the accuracy of DFTB compared to DFT on much smaller antidot lattices in terms of the band structures. ${ }^{34}$ Since geometry relaxation is the most costly task in DFT we also investigate the cost benefits of combining the two meth- ods. By using DFT and elaborating on the role of spin, we also wish to address some of the main features found specifically for antidot lattices on a tight-binding level of the theory.

This paper is organized as follows. In Sec. II, we introduce the antidot lattice systems and the methods used. The equilibrium geometries and band structures obtained using both DFT and DFTB and a combination thereof is given in Sec. III together with a detailed investigation of the spin properties. We conclude with a short summary.

\section{SYSTEMS AND METHOD}

The specific realization of the antidot lattice we consider in this paper is a hexagonal (triangular) array of holes in a graphene sheet as proposed by Pedersen et al. ${ }^{9}$ Within the hexagonal unit cells there can be different hole geometries, and two examples of high symmetry holes are shown in Fig. 1. Below, geometries are fully relaxed but as a starting point ideal geometries using fixed bond lengths and angles of $120^{\circ}$ are constructed. These geometries furthermore provide a straightforward notation for the structures. Thus, we designate antidot lattices with circular holes according to the notation $\{L, R\}_{\bigcirc}$, where $L$ is the side length of the unit cell and $R$ the hole radius, both measured in units of the graphene lattice constant $a=2.46 \AA$ giving a C-C bond length of $1.42 \AA$. Similarly, for triangular holes, we apply the notation $\{L, D\}_{\triangle}$, where $D$ denotes the side length of the hole. ${ }^{19}$ The holes are passivated with $\mathrm{H}$ using a $\mathrm{C}-\mathrm{H}$ bond length of $1.1 \AA$ and consist almost entirely of zigzag edges. These structures are idealized but may well be within experimental reach given the recent advancements. ${ }^{17,18}$

For the first principles calculations we have used the $a b$ initio pseudopotential DFT as implemented in the SIESTA code $\mathrm{e}^{35}$ to obtain the electronic structure and relaxed atomic positions from spin-polarized DFT. ${ }^{36} \mathrm{We}$ employ the generalized gradient approximation (GGA) Perdew-BurkeErnzerhof (PBE) functional for exchange correlation. ${ }^{37}$

In the DFTB methodology, the Kohn-Sham orbitals are expanded in a set of nonorthogonal tight-binding orbitals. This basis along with all two-center integrals is obtained from a self-consistent DFT calculation for the dimer, i.e., for $\mathrm{C}-\mathrm{C}, \mathrm{H}-\mathrm{H}$, and $\mathrm{C}-\mathrm{H}$ pairs in our case. In the present work, we use the original $(\mathrm{C}, \mathrm{H})$ parametrization of Porezag et al. ${ }^{33}$ which does not include spin. Once all required hopping and overlap matrix elements are tabulated as a function of interatomic distance, the electronic band structure is easily obtained from a generalized eigenvalue problem. To relax geometries, however, the total energy of trial geometries must be computed. This goes beyond ordinary tight-binding schemes. In DFTB, the total energy is written as the sum

$$
E_{\mathrm{tot}}=E_{\mathrm{bs}}+\sum_{n<m} V_{\mathrm{rep}}\left(r_{n m}\right)
$$

where $E_{\mathrm{bs}}$ is the band structure energy, i.e., the sum of energy eigenvalues for all occupied states, and $V_{\text {rep }}$ is the so-called repulsive potential. The repulsive energy is approximated as a sum over atomic pairs $n, m$ with separation $r_{n m}$. Similarly to the two-center integrals, the repulsive potential is deter- 
TABLE I. The band gaps for various systems calculated with either DFT or DFTB using geometries obtained with different methods for relaxation. All values are in $\mathrm{eV}$.

\begin{tabular}{|c|c|c|c|c|c|c|}
\hline \multirow[b]{2}{*}{ Relaxation } & \multicolumn{3}{|c|}{ DFT } & \multirow{2}{*}{$\begin{array}{c}\text { Nonspin DFT } \\
\text { DFTB }\end{array}$} & \multicolumn{2}{|c|}{ DFTB } \\
\hline & None & DFT & DFTB & & None & DFTB \\
\hline$\{4,2\}_{\bigcirc}$ & 0.93 & 1.01 & 0.97 & 0.97 & 0.99 & 1.05 \\
\hline$\{5,2.8\}_{\bigcirc}$ & 0.72 & 0.84 & 0.79 & 0.79 & 0.88 & 0.98 \\
\hline$\{5,3.5\}_{\bigcirc}$ & 1.27 & 1.51 & 1.35 & 1.35 & 1.72 & 1.74 \\
\hline$\{6,3.6\}_{\bigcirc}$ & 0.39 & 0.52 & 0.46 & 0.46 & 0.65 & 0.75 \\
\hline$\{6,5\}_{\triangle}$ & 0.24 & 0.22 & 0.22 & 0.00 & 0.00 & 0.00 \\
\hline
\end{tabular}

mined by equating Eq. (1) to the DFT total energy for suitable reference systems taken to be the above dimers as well as graphite. ${ }^{33}$ In this manner, geometry relaxation becomes feasible in a tight-binding scheme with only a small computational cost. ${ }^{38}$

DFTB is thus a considerable simplification of DFT why the latter is the most precise description.

\section{RESULTS}

For all considered structures a structural relaxation with DFT leads to a shrinking of the hole, of the order of $1 \%$, resulting in $\mathrm{C}-\mathrm{C}$ bonds close to the edges stretching and contracting in the range of $1.39-1.45$ (1.43 for $\left.\{5,3.5\}_{\circ}\right) \AA$. A few bond lengths away from the hole edge the $\mathrm{C}-\mathrm{C}$ bond length remains unaltered at $1.42 \AA$.

In the case of relaxation with DFTB the picture is quite similar. Edge-atom C-C bond lengths vary from $1.39-1.42 \AA$ for all systems but $\{6,5\}_{\triangle}$, which has variations of $1.38-1.44 \AA$. The shrinking of the hole size is smaller than $1 \%$.

The results for the band gaps for five different systems are summarized in Table I. The band structures are calculated using both DFT and DFTB on structures relaxed at different accuracy levels and thus at different computational costs. The combinations are not exhaustive but represent a relevant set aimed at saving computational costs. The relaxation type is given in the second row of Table I. DFTB is expected to match DFT better as $L / R$ increases due to decreased importance of edge details. The systems chosen here are mostly edge-dominated (low $L / R$ ratio) and thus represent the worst case scenario.

Using DFT we find band gaps ranging from 0.2 to $1.5 \mathrm{eV}$ confirming that the antidot lattice turns the semimetallic graphene into a semiconductor. ${ }^{9}$ However, only spinpolarized DFT predicts a band gap for the $\{6,5\}_{\triangle}$ structure, which will be discussed in detail below.

Pedersen et al. ${ }^{9}$ demonstrated a scaling law between the hole size and the band gap for large $L / R$ ratios but no such simple picture for small $L / R$ ratios emerged. This trend agrees well with our edge-dominated systems were no simple scaling between the hole size and the band gap could be identified.

As illustrated on Fig. 2, DFTB in general gives a larger band gap than DFT (the circles lie consistently above the dashed line). This tendency is enhanced the more edgedominated the structure becomes. On average, the DFTB gap is $20 \%$ larger than the DFT value for the four structures with circular perforations. The discrepancy increases for structures with holes occupying a large portion of the unit cell, such as $\{5,3.5\}_{\bigcirc}$. Moreover, for $\{6,5\}_{\triangle}$ the omission of spin effects leads incorrectly to a vanishing DFTB band gap in agreement with nonspin DFT. In the cases of the $\{4,2\} \bigcirc$ and $\{6,5\}_{\triangle}$ systems the band structures are shown in Fig. 3 left and right, respectively, calculated using DFT (DFTB) in the upper(lower) panel. We see that the shape of the bands agrees qualitatively for the two methods.

\section{A. Relaxation}

We next analyze the importance of relaxation. The clear trend is that relaxation increases the band gap. This is illustrated for DFT in Fig. 2 (crosses corresponding to the unrelaxed structure lie below the dotted line), as well as in Table I for DFTB. Comparing unrelaxed results with fully relaxed results we see from Table I a change in band gaps within $10 \%$ and $15 \%$ using DFTB and DFT, respectively. Only in the case of DFT does the effect of relaxation increase the

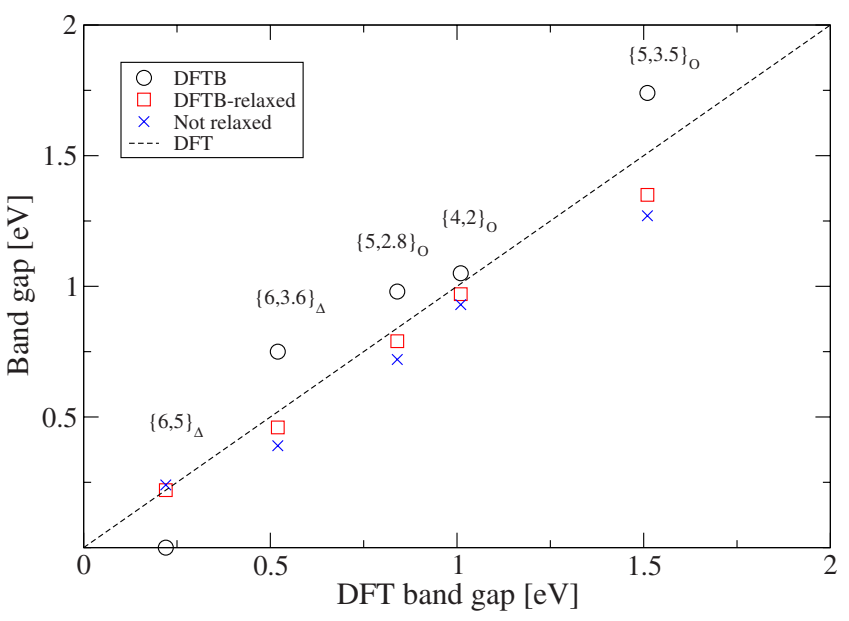

FIG. 2. (Color online) The band gaps for DFTB, DFT on DFTBrelaxed geometry, and DFT on unrelaxed geometry plotted versus pure DFT results. Points above (below) the dotted line are thus overestimated (underestimated) compared to pure DFT. Note, that DFTB calculates the electronic structure without spin and fails to predict a band gap for the $\{6,5\}_{\triangle}$ structure. 

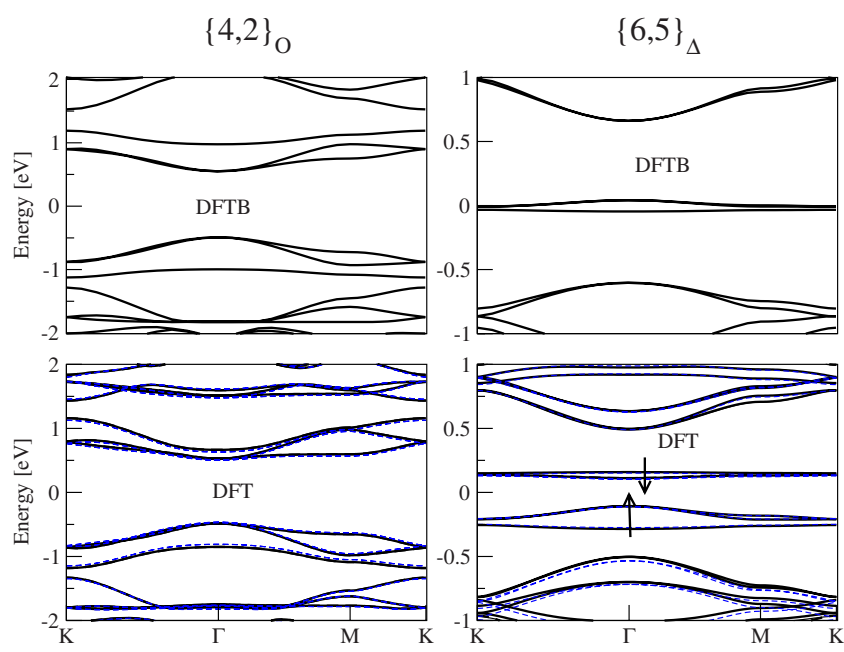

FIG. 3. (Color online) Band structures for the $\{4,2\}_{\bigcirc}$ (left column) and $\{6,5\}_{\triangle}$ (right column) systems using DFTB (upper panels) and DFT (lower panels). The dotted blue/dark gray curves in the lower panels are DFT results using structures relaxed with DFTB. The arrow pointing up (down) indicates that bands are filled with majority (minority) electrons only.

more edge-dominated the system becomes. The change using DFT for the $\{4,2\}_{\bigcirc}$ system is $8 \%$ compared to $16 \%$ for $\{5,3.5\}_{\bigcirc}$.

It must be emphasized that larger differences between initial and relaxed geometries may well give rise to a larger discrepancy between their band gaps. However, even for the case of a single passivated edge-defect the difference in relaxed and unrelaxed DFT band gaps is less than $10 \%$.

By relaxing the geometry with DFTB the DFT results are improved from the non-relaxed case as shown in Fig. 2 (the squares are closer to the dashed line than the crosses). Compared to pure DFT the largest difference in the band gap is again found for the edge-dominated $\{5,3.5\}_{\circ}$ system: it is now $11 \%$ compared to $16 \%$ without relaxation. For the larger $\{6,5\}_{\triangle}$ structure we find the same values as for pure DFT. The DFT results are shown for both DFT-relaxed and DFTBrelaxed structures on Fig. 3 indicated by thick and dotted line, respectively. The different geometries do not change the bands notably.

Using DFT on DFTB-relaxed structures is thus an approach with a good trade off between accuracy and computational cost. This finding is of great practical use, since relaxation is very costly in DFT.

\section{B. Magnetic properties}

For the $\{6,5\}_{\triangle}$ system with both non-spin-DFT and DFTB there are three (one nearly doubly degenerate) bands with weak dispersion at zero energy. Introducing spin leads to a clear splitting of these bands, i.e., to the formation of a band gap. For a comparison of DFT and DFTB, see Fig. 3, right column. The three bands below (above) the Fermi level are half-filled by majority (minority) spin electrons and are thus completely spin-polarized. The size of the band gap is thus also an indication of the robustness of the magnetic state. $^{39}$
The magnetic moments of the structures can be understood as a consequence of graphene being a bipartite lattice in the nearest neighbor approximation as shown by Lieb. ${ }^{20}$ According to Lieb's theorem, ${ }^{20}$ the total magnetic moment can be written as $M=N_{A}-N_{B}$ where $N_{A(B)}$ is the number of atoms occupying the $A(B)$ sites of the bipartite graphene lattice. Thus, if the angle between the zigzag edges is $0^{\circ}$ or $60^{\circ}$ the edge-atoms belong to the same sublattice, while they belong to different sublattices if the angle is $120^{\circ}$ or $180^{\circ}$. Consequently, the hexagonal hole is non-magnetic and the triangular is magnetic. This is consistent with a Mulliken analysis from the DFT calculations which shows a nonzero magnetic moment only for the $\{6,5\}_{\triangle}$ system of $3.00 \mu_{B}$ per unit cell. By inspection of the geometry we indeed find $N_{A}$ $-N_{B}=3$.

As a continuation of Lieb's work, Inui et al. ${ }^{28}$ showed that such sublattice imbalance results in there being $N_{A}-N_{B}$ midgap states with zero energy. We, thus, expect a degeneracy of 3 of the low-dispersion bands in the $\{6,5\}_{\triangle}$ case.

In Fig. 3, lower right panel, there is one largely dispersionless band just below two almost completely degenerate bands which have some dispersion especially at the $\Gamma$-point. Such band curvature was also found for hydrogenated graphene ribbons by Kusakabe et al. ${ }^{39}$ The $\Gamma$-point states for each band are shown in Fig. 4, where the strong localization is seen for the lowest band, Fig. 4(c), whereas the bands with curvature, Figs. 4(a) and 4(b), yield less localized states. Note also the alternation in the amplitudes of the states between sublattices as proposed by Inui et al. ${ }^{28}$ The state of the highest occupied (spin-degenerate) band for the nonmagnetic $\{6,3.6\} \bigcirc$ structure is shown for comparison in Fig. 4(d). The splitting as well as the curvature is less pronounced for the unfilled states above the Fermi level showing particle-hole asymmetry. This asymmetry is expected due to the breaking of the symmetry of the bipartite lattice partly due to the DFT treatment beyond nearest neighbor as well as the passivation of the edges which changes the on-site potential at edge sites. This is inherently also the case for DFTB. By inspection of the SIESTA Hamiltonian ${ }^{35}$ we find an increase in on-site energy for passivated edge atoms as compared to atoms far from the edge. Vanevic et al. ${ }^{19}$ find that a potential shift on the edge atoms mainly causes a lifting of the degeneracy of the flat bands, consistent with our observations.

As mentioned above, the global spin is given by the sublattice imbalance. This does not, however, determine the local spin. For the hexagonally shaped hole structures we find not only a zero global spin, but also a zero spin on all atoms. This explains the identical band gaps found using DFT with and without spin. Such nonmagnetic solutions have been found for finite graphene ribbons or graphene flakes by Jiang et al. ${ }^{24}$ using DFT. They find a sudden transition from nonmagnetic to magnetic solutions going from a system size of $[3,3]$ and $[4,3]$ with numbers indicating rings in the graphene lattice along zigzag and armchair directions. Such transitions are also seen in slits cut in graphene in a study by Kumazaki et $a .^{23}$ Viewing each edge in our hexagonal structures as ribbons, the largest ribbon corresponds to a $[4,3]$ ribbon $\left(\{6,3.6\}_{\circ}\right.$ structure). We, thus, expect local magnetization to appear for slightly larger systems.

For $\{6,5\}_{\triangle}$ we have the strongest polarization at the middle of each edge with maximum magnetic moment per 

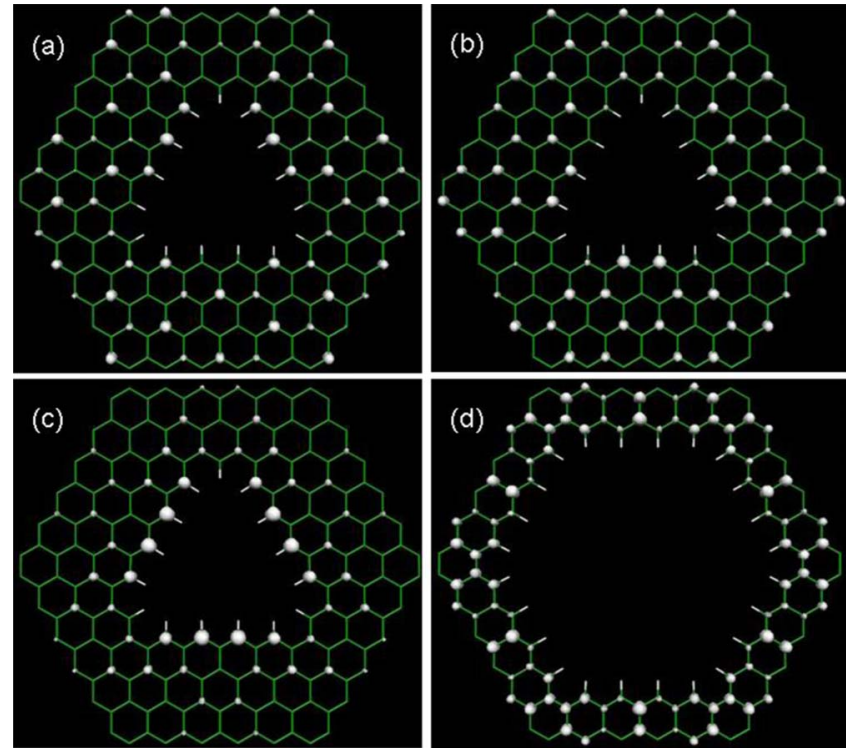

FIG. 4. (Color online) Amplitudes of certain important states. $(a, b)$ : degenerate midgap states, (c): nondegenerate midgap state of $\{6,5\}_{\triangle},(\mathrm{d})$ : highest filled bands of $\{6,3.6\}_{\bigcirc}$. All states are calculated at the $\Gamma$ point.

atom being $0.24 \mu_{B}$. We note that each corner atom has a magnetization $-0.03 \mu_{B}$. The edge-atom magnetization is below the maximum of $1 / 3 \mu_{B}$ for graphene ribbons when the width becomes too large for edge-edge interactions. Our systems thus have edge-edge interactions, which is expected due to the ribbon width of 6 rings. A plot of the spinpolarized density ${ }^{40}$ is shown in Fig. 5. The majority spins reside mostly on the edges of the dominant sublattice sites. Neighboring sites on the other sublattice have minority spin polarization. Note also that the nonzero spin of the atoms far from the edges indicating interaction between neighboring hole edges.

\section{CONCLUSION}

Using DFT and DFTB we have calculated band gaps in various antidot lattice geometries. The computed band gaps range from 0.2 to $1.5 \mathrm{eV}$. In general, DFTB gives larger band gaps than DFT with the largest difference for nonmagnetic structures of $44 \%$. Geometry relaxation using either method is found to increase the band gap with maximally $15 \%$. Combining the two methods by performing a DFT calculation on a DFTB-relaxed structure is found to give a good trade off

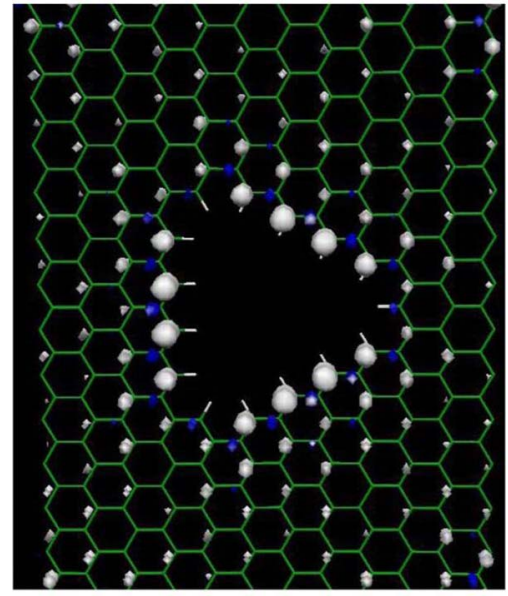

FIG. 5. (Color online) The difference in majority spin and minority spin densities for the $\{6,5\}_{\triangle}$ structure. Blue/dark gray (white) indicates surplus of majority(minority) spin.

between accuracy and computational cost facilitating the treatment of larger systems. However, even for unrelaxed geometries we find qualitative agreement with the DFTrelaxed geometries. Trends for ideal geometries as presented here can thus be investigated without any relaxation in the nonmagnetic case.

Certain geometries are shown with DFT to have a nonzero total magnetic moment which is understood via Lieb's theorem as a consequence of sublattice imbalance. For these structures a spin-polarized treatment is needed to achieve even qualitative results for the band gaps. Local spin, which can occur regardless of the total magnetic moment, is not observed for very edge-dominated systems. Sublattice imbalance leads to the occurrence of low-dispersion midgap bands. We find a lifting of degeneracy of these otherwise degenerate bands on a perfect bipartite lattice as well as a considerable spin-splitting. These completely spin-polarized states are primarily located at the hole edges.

\section{ACKNOWLEDGMENTS}

We thank Jesper G. Pedersen for fruitful discussions. Financial support from Danish Research Council FTP grant "Nanoengineered graphene devices" is gratefully acknowledged. Computational resources were provided by the Danish Center for Scientific Computations (DCSC). A.P.J. is grateful to the FiDiPro program of the Finnish Academy.

\footnotetext{
*Corresponding author; joachim.fuerst@ nanotech.dtu.dk

${ }^{1}$ A. Geim and K. Novoselov, Nature Mater. 6, 183 (2007).

${ }^{2}$ M. I. Katsnelson, K. S. Novoselov, and A. K. Geim, Nat. Phys. 2, 620 (2006).

${ }^{3}$ P. Avouris, Z. Chen, and V. Perebeinos, Nat. Nanotechnol. 2, 605 (2007).

${ }^{4}$ K. S. Novoselov, A. K. Geim, S. V. Morozov, D. Jiang, M. I.
}

Katsnelson, I. V. Grigorieva, S. V. Dubonos, and A. A. Firsov, Nature (London) 438, 197 (2005).

${ }^{5}$ K. Novoselov and A. Geim, Mater. Technol. 22, 178 (2007).

${ }^{6}$ M. Y. Han, B. Ozyilmaz, Y. B. Zhang, and P. Kim, Phys. Rev. Lett. 98, 206805 (2007).

${ }^{7}$ M. D. Fischbein and M. Drndi'c, Appl. Phys. Lett. 93, 113107 (2008). 
${ }^{8}$ K. S. Kim, Y. Zhao, S. Y. Lee, J. M. Kim, K. S. Kim, J.-H. Ahn, P. Kim, J.-Y. Choi, and B. H. Hong, Nature (London) 457, 706 (2009).

${ }^{9}$ T. G. Pedersen, C. Flindt, J. Pedersen, N. A. Mortensen, A. P. Jauho, and K. Pedersen, Phys. Rev. Lett. 100, 136804 (2008).

${ }^{10}$ T. G. Pedersen, C. Flindt, J. Pedersen, A. P. Jauho, N. A. Mortensen, and K. Pedersen, Phys. Rev. B 77, 245431 (2008).

${ }^{11}$ J. Eroms and D. Weiss, arXiv:0901.0840 (unpublished).

${ }^{12}$ T. Shen, Y. Q. Wu, M. A. Capano, L. P. Rokhinson, L. W. engel, and P. D. Ye, Appl. Phys. Lett. 93, 122102 (2008).

${ }^{13}$ J. H. Chen, W. G. Cullen, C. Jang, M. S. Fuhrer, and E. D. Williams, Phys. Rev. Lett. 102, 236805 (2009).

${ }^{14}$ Y. Zhang, T. T. Tang, C. Girit, H. Zhao, M. C. Martin, A. Zettl, M. F. Crommie, Y. R. Shen, and F. Wang, Nature (London) 459, 820 (2009).

${ }^{15}$ T. G. Pedersen, A. P. Jauho, and K. Pedersen, Phys. Rev. B 79, 113406 (2009).

${ }^{16}$ K. A. Ritter and J. W. Lyding, Nature Mater. 8, 235 (2009).

${ }^{17}$ C. O. Girit, J. C. Meyer, R. Erni, and M. D. Rossel, Science 323, 1705 (2009).

${ }^{18}$ X. Jia et al., Science 323, 1701 (2009).

${ }^{19}$ M. Vanevic, V. M. Stojanovic, and M. Kindermann, Phys. Rev. B 80, 045410 (2009).

${ }^{20}$ E. H. Lieb, Phys. Rev. Lett. 62, 1201 (1989).

${ }^{21}$ P. O. Lehtinen, A. S. Foster, Y. Ma, A. V. Krasheninnikov, and R. M. Nieminen, Phys. Rev. Lett. 93, 187202 (2004).

22 J. J. Palacios, J. Fernandez-Rossier, and L. Brey, Phys. Rev. B 77, 195428 (2008).

${ }^{23}$ H. Kumazaki and D. S. Hirashima, Low Temp. Phys. 34, 805 (2008).

${ }^{24}$ D. E. Jiang, B. G. Sumpter, and S. Dai, J. Chem. Phys. 127, 124703 (2007).

${ }^{25}$ M. R. Philpott, F. Cimpoesu, and Y. Kawazoe, Chem. Phys. 354, 1 (2008).

${ }^{26}$ O. V. Yazyev and L. Helm, Phys. Rev. B 75, 125408 (2007).

${ }^{27}$ M. Fujita, K. Wakabayashi, K. Nakada, and K. Kusakabe, J. Phys. Soc. Jpn. 65, 1920 (1996).

${ }^{28}$ M. Inui, S. A. Trugman, and E. Abrahams, Phys. Rev. B 49,
3190 (1994).

${ }^{29}$ D. Bahamon, A. L. C. Pereira, and P. A. Schulz, Phys. Rev. B 79, 125414 (2009).

${ }^{30}$ Y. Hancock, K. Saloriutta, A. Uppstu, A. Harju, and M. Puska, J. Low Temp. Phys. 153, 393 (2008).

${ }^{31}$ D. Yu, E. Lupton, M. Liu, and F. Liu, Nano Res. 1, 56 (2008).

${ }^{32}$ Y. P. Chen, Y. E. Xie, and X. H. Yan, J. Appl. Phys. 103, 063711 (2008).

${ }^{33}$ D. Porezag, T. Frauenheim, T. Kohler, G. Seifert, and R. Kaschner, Phys. Rev. B 51, 12947 (1995).

${ }^{34}$ We note, that DFT is known to underestimate the band gap, see, e.g., M. S. Hybertsen and S. G. Louie, Phys. Rev. B 34, 5390 (1986).

${ }^{35}$ J. M. Soler, E. Artacho, J. D. Gale, A. Garcia, J. Junquera, P. Ordejon, and D. Sanchez-Portal, J. Phys.: Condens. Matter 14, 2745 (2002).

${ }^{36}$ The systems are relaxed using the conjugate gradient $(\mathrm{CG})$ method with a force tolerance of $0.01 \mathrm{eV} / \AA$. All atoms are allowed to move during relaxation but the unit cell is kept fixed with a vacuum between graphene layers in neighboring cells of $10 \AA$. The mesh cutoff value defining the real space grid used is 175 Ry and we employ a double- $\zeta$ polarized (DZP) basis set. A Monkhorst-Pack grid of $(2,2,1)$ was found sufficient for all structures.

${ }^{37}$ J. P. Perdew, K. Burke, and M. Ernzerhof, Phys. Rev. Lett. 77, 3865 (1996).

${ }^{38}$ We relax the two outermost rows of atoms along the perimeter of the hole until the total energy per atom was converged to better than $10^{-4} \mathrm{eV}$.

${ }^{39}$ K. Kusakabe and M. Maruyama, Phys. Rev. B 67, 092406 (2003).

${ }^{40}$ Due to the larger cell used in this calculation we use less accurate settings (single- $\zeta$ basis, 100 Ry mesh, one $k$ point). This changes the magnitude of the spin somewhat as compared to the smaller cell calculations. The maximum magnetic moment per atom is $0.27 \mu_{B}$ for the large cell versus $0.24 \mu_{B}$ for the smaller. The qualitative picture of the spin distribution is, however, the same for both calculations. 